\title{
Hierarquia das Necessidades de Maslow: Validação de um Instrumento
}

\author{
Thiago Medeiros Cavalcanti ${ }^{1}$ \\ ${ }^{1}$ Universidade Federal da Paraíba, PB, Brasil. \\ Emerson Diógenes de Medeiros ${ }^{2}$ \\ ${ }^{2}$ Universidade Federal do Piauí, PI, Brasil. \\ Hysla Magalhães de Moura ${ }^{3}$ \\ ${ }^{3}$ Universidade do Estado do Rio de Janeiro, RJ, Brasil.
}

\author{
Valdiney Veloso Gouveia ${ }^{1}$ \\ ${ }^{1}$ Universidade Federal da Paraíba, PB, Brasil. \\ Tailson Evangelista Mariano ${ }^{1}$ \\ ${ }^{1}$ Universidade Federal da Paraíba, PB, Brasil. \\ Heloísa Bárbara Cunha Moizeís ${ }^{1}$ \\ ${ }^{1}$ Universidade Federal da Paraíba, PB, Brasil.
}

Resumo: Este estudo teve como objetivo adaptar ao contexto brasileiro o Inventário de Satisfação das Necessidades Básicas (ISNB), reunindo evidências de sua validade (fatorial e convergente) e consistência interna. Realizaram-se dois estudos [Estudo $1(n=200)$ e Estudo 2 ( $\mathrm{n}=199)$ ], contando com a participação de estudantes universitários, que responderam o ISNB e perguntas demográficas; no Estudo 1 eles também responderam a Escala de Satisfação com a Vida. Os resultados da análise fatorial exploratória (Estudo 1) indicaram um modelo com cinco ou seis fatores, porém se optou pelo primeiro por ser mais parcimonioso e coerente com a teoria. Os cinco fatores se correlacionaram positivamente com a satisfação com a vida. Posteriormente, realizou-se uma análise fatorial confirmatória (Estudo 2) em que se compararam os modelos uni, penta e hexafatorial, tendo o de cinco fatores se revelado mais adequado. Em ambos os estudos os alfas de Cronbach dos cinco fatores se mostraram adequados para fins de pesquisa $(\alpha>0,60)$. Concluiu-se que o ISNB é uma medida psicometricamente adequada, podendo ser empregada em contexto brasileiro para conhecer a satisfação das necessidades das pessoas e seus correlatos.

Palavras-chave: Necessidades, Maslow, Inventário, Validade, Precisão.

\section{Maslow's Hierarchy of Needs: Validation of an Instrument}

Abstract: This study aimed to adapt the Basic Needs Satisfaction Inventory (BNSI) to the Brazilian context, gathering evidence of its validity (factorial and convergent) and reliability. Two studies were carried out [Study $1(n=200)$ and Study $2(n=199)$ ], considering as participants undergraduate students. They answered the BNSI and demographic questions; in Study 1 they also answered the Life Satisfaction Scale. Results of the exploratory factorial analysis (Study 1) indicated a model with five or six factors, but the first solution was chosen because it was more parsimonious and in line with the theory. All five factors correlated positively with satisfaction with life. Subsequently, a confirmatory factorial analysis was performed (Study 2) in which the one, five and six-factors models were compared, with five factors being more adequate. In both studies Cronbach's alphas of the five factors proved to be adequate for research purposes $(\alpha>0.60)$. In conclusion, the BNSI is a psychometrically adequate measure and can be used in a Brazilian context to know the people's satisfaction of needs and their correlates.

Keywords: Needs, Maslow, Inventory, Validity, Reliability. 


\title{
Jerarquía de Necesidades de Maslow: Validación de un Instrumento
}

\begin{abstract}
Resumen: Este estudio tuvo como objetivo adaptar el Inventario de Satisfacción de Necesidades Básicas (ISNB) al contexto brasileño, reuniendo evidencia de su validez (factorial y convergente) y consistencia interna. Se llevaron a cabo dos estudios [Estudio $1(n=200) y$ Estudio 2 ( $\mathrm{n}=199$ )], con la participación de estudiantes universitarios, que respondieron al ISNB y preguntas demográficas; en el Estudio 1 también respondieron la Escala de Satisfacción con la Vida. Los resultados del análisis factorial exploratorio (Estudio 1) indicaron un modelo con cinco o seis factores, pero el primero fue elegido para ser más parsimonioso y consistente con la teoría. Los cinco factores se correlacionaron positivamente con la satisfacción con la vida. Posteriormente, se realizó un análisis factorial confirmatorio (Estudio 2) comparando los modelos uni, penta y hexafactorial, y el modelo de cinco factores fue más apropiado. En ambos estudios, los alfa de Cronbach de los cinco factores demostraron ser adecuados para fines de investigación $(\alpha>0,60)$. Se concluyó que el ISNB es una medida psicométrica adecuada y puede usarse en un contexto brasileño para conocer la satisfacción de las necesidades de las personas y sus correlatos.
\end{abstract}

Palabras clave: Necesidades, Maslow, Inventario, Validez, Precisión.

\section{Introdução}

As necessidades humanas se inserem no contexto das teorias motivacionais (Gouveia, 2013; Maslow, 1943), elaborando-se modelos que variam segundo o contexto ou a área de atuação (por exemplo, clínico, educacional, organizacional), a corrente em que estão fundamentados (por exemplo, psicanálise, comportamentalismo, humanismo) e a sua origem admitida (apreendida ou inata). A propósito da diversidade de modelos, parece bastante aceita a proposta elaborada por Maslow (1954), que apresentou uma teoria que pressupunha uma hierarquia das necessidades, tendo impacto destacado na Psicologia (Kenrick, Griskevicius, Neuberg, \& Schaller, 2010). Apesar de sua relevância, esta teoria não esteve imune a críticas, a exemplo da própria organização hierárquica das necessidades ou o fato de ter sido desenvolvida em contexto cultural específico, isto é, Estados Unidos, considerando uma perspectiva eminentemente clínica (Yang, 2003).

A propósito da teoria de Maslow, ele sugere que a vida motivacional pode ser descrita como uma escalada por diferentes níveis de uma pirâmide que estrutura as necessidades. Por exemplo, no nascimento os bebês são motivados por necessidades mais básicas, como as fisiológicas; na sequência as necessidades de segurança prevalecem e, segundo crescessem, têm lugar as necessidades de filiação e, mais tarde, aquelas de estima. Segundo este autor, é a partir do início da adolescência quando os indivíduos já podem ser conduzidos em direção à autorrealização, que se consolida naquelas pessoas mais maduras (Maslow, 1954). Apesar do anteriormente comentado, isso não significa que todos os adultos são motivados à autorrealização ou a alcançam. Em qualquer caso, este autor pressupõe que, caso alguma necessidade básica seja frustrada, isso dará lugar a psicopatologias.

Considerando que a teoria maslowniana é recorrente na literatura, recebendo suporte de várias pesquisas empíricas (Kellerman, 2014; Lenthe, Jansen, \& Kamphuis, 2015; Toarmina \& Gao, 2013; Tay, \& Diener, 2011), justifica-se a atenção que aqui se dispensa a ela. Isso é reforçado em razão de seu uso e aplicação no contexto brasileiro ser ainda precário, sobretudo em razão de não contar com um instrumento psicometricamente adequado para medir as necessidades propostas por Maslow (1954). A propósito, o presente artigo procura oferecer uma contribuição nesta direção, adaptando ao contexto brasileiro o Inventário de Satisfação das Necessidades Básicas (ISNB) (Leidy, 1994), reunindo evidências de sua validade fatorial, validade convergente e consistência interna. Antes de considerá-lo mais detalhadamente, demanda-se aclarar as necessidades fundamentais na tipologia maslowniana. 


\section{Definição de necessidades}

De acordo com a abordagem de Maslow (1954), as necessidades humanas são classificadas em cinco categorias principais: fisiológicas, segurança, afiliação (pertencimento), estima e autorrealização. Conforme se indicou anteriormente, admite-se que tais necessidades surgem sequencialmente, desenvolvendo-se desde o nascimento até a idade adulta. Assim, as mais básicas, o alicerce da pirâmide hierárquica (por exemplo, necessidades fisiológicas), possuem maior prevalência no início do desenvolvimento que aquelas mais elevadas na hierarquia (por exemplo, autorrealização). Estima-se que quanto maior o grau de satisfação das necessidades, melhor a saúde mental do indivíduo (Lester, 2013). A seguir são descritas essas necessidades:

Necessidade fisiológica. É a base da pirâmide, surgindo desde o início do desenvolvimento humano. Pode ser definida com a ausência de substâncias químicas e nutrientes para o organismo, ou em razão de condições ambientais adversas que podem colocar o corpo em risco, a exemplo do frio extremo (Toarmina, \& Gao, 2013). Em termos empíricos, Tay e Diener (2011), ao realizarem uma pesquisa em 155 países, encontraram uma associação forte entre as necessidades mais básicas e a sociedade em que as pessoas vivem, isto é, quanto mais carente o país em que os participantes viviam, maior a pontuação nesta necessidade. $\mathrm{O}$ mesmo resultado não se repetiu com as outras necessidades da pirâmide.

Necessidade de segurança. Este tipo de necessidade é fundamental para garantir a sobrevivência dos seres humanos, sendo definida como a falta de proteção. Pode ser observada quando ocorre uma reação ao perigo evidente e a estímulos ameaçadores, sendo esta reação considerada instintiva (Maslow, 1943). De acordo com Carretié, Hinojosa, Mercado e Tapia (2005), respostas instintivas são geneticamente determinadas. Exemplos de elementos que asseguram a satisfação da necessidade de segurança são ter uma casa segura, contar com uma figura protetora (por exemplo, pais, polícia), viver em um sistema jurídico confiável e contar com estabilidade na vida (Toarmina, \& Gao, 2013).

Necessidade de afiliação. É a categoria intermediária na hierarquia das necessidades. Diferentemente das anteriores, não é considerada por Maslow como fundamental para a sobrevivência do indivíduo; no caso, refere-se às relações interpessoais, ao sentimento de pertença a grupos sociais e às díades íntimas em que existe a preocupação afetiva mútua. Kenrick et al. (2010) defendem a base evolutiva das necessidades de afiliação, indicando que os grupos humanos envolvem extensa partilha de recursos, conhecimento e tarefas parentais, favorecendo a sobrevivência de seus membros. Neste sentido, estima-se que a rejeição social produza respostas que utilizam os mesmos circuitos neurais envolvidos na dor física (MacDonald, \& Leary, 2005).

Necessidades de estima. Maslow (1943) dividiu esta necessidade em dois componentes: estima de si e a estima recebida dos outros. O primeiro está relacionado com o respeito que a pessoa atribui para si mesmo, o mérito de suas conquistas e a dignidade de sua vida. O segundo, por sua vez, diz respeito ao desejo de boa reputação, status, dominância e glória recebida dos outros. De forma geral, a necessidade de estima se torna saliente quando ocorre falta de respeito de si mesmo e/ou a falta de respeito que o indivíduo recebe de outras pessoas (Toarmina, \& Gao, 2013).

Necessidade de autorrealização. É o nível mais elevado na hierarquia das necessidades, representando a tendência de as pessoas se tornarem aquilo que potencialmente estão destinadas a ser. Portanto, implica realizar suas capacidades e seus talentos, cumprindo com suas vocações. Neste sentido, podem ser identificadas como características de pessoas autorrealizadas a espontaneidade, a criatividade, a autonomia e a resistência à doutrinação; destacam-se, ainda, a identificação com a espécie humana como um todo e a maior aceitação de si mesmo, dos outros e da natureza (Maslow, 1943).

De acordo com Maslow (1954), apenas as necessidades que não são satisfeitas atuam como força motivadora. Quando é gratificada ou atendida, a necessidade consecutiva na hierarquia passa a determinar o comportamento do indivíduo. A propósito, a gratificação de uma necessidade muda os processos cognitivos bem como as atitudes e a avaliação dos objetos e situações (Toarmina, \& Gao, 2013). Portanto, o sistema de necessidades é influenciado por duas forças: a privação e a gratificação. A privação, entendida como falta de satisfação de uma necessidade específica, conduz à prevalência de comportamentos no indivíduo para satisfazê-la, isto é, o desempenho acaba sendo motivado por elas. A gratificação de uma necessidade faz com que aquela de um nível superior 
assuma a importância, suscitando a dominância de outros comportamentos até que esta seja também gratificada ou satisfeita (Furnham, 2005). Portanto, o processo funciona como segue: privação do nível baixo $\otimes$ dominância de comportamentos $\otimes$ gratificação $\otimes$ ativação do nível superior.

Quanto ao conceito de emergência de uma nova necessidade, após a satisfação da anterior, ela não ocorrerá de forma súbita, mas gradual. A título de ilustração, considere-se o que Maslow (1954) comenta sobre a satisfação das necessidades, evidenciando que pode não ser absoluta ou ocorrer abruptamente: "o cidadão médio é satisfeito talvez $85 \%$ em necessidades fisiológicas, $70 \%$ em necessidade de segurança, $50 \%$ em necessidade de afiliação, $40 \%$ em necessidade de estima e $10 \%$ em necessidade de autorrealização" (p. 54). Deste modo, a satisfação de uma necessidade aos poucos vai dando margem ao aparecimento de outras imediatamente superiores.

Em resumo, parece plausível o conjunto de necessidades postuladas por Maslow, que vem encontrando correspondência em outros modelos. Embora sua concepção sobre a hierarquia das necessidades suscite algumas dúvidas, principalmente no que diz respeito à transição de um nível para o outro, ter a chance de medir as necessidades que este autor pressupõe parece fundamental para entender seus correlatos, auxiliando em temáticas afins, a exemplo dos valores, interesses vocacionais ou traços de personalidade (Gouveia, 2013; Maslow, 1954). Partindo deste entendimento, considerando que não se encontrou qualquer medida dessas necessidades na realidade brasileira, decidiu-se adaptar para este contexto o ISNB (Leidy, 1994), que se descreve a seguir.

\section{Inventário de Satisfação das Necessidades Básicas}

Abraham Maslow não chegou a desenvolver um instrumento para avaliar as necessidades humanas, o que se constituiu em um entrave para testar alguns de seus pressupostos. Não obstante, esta lacuna foi posteriormente preenchida por Leidy (1994), quem desenvolveu o Basic Need Satisfaction Inventory (BNSI). Os itens desta medida foram derivados dos 123 indicadores subjetivos apresentados por Andrews e Withey (1974), que tiveram em conta um conjunto com mais de 800 itens elaborados com base em entrevistas estruturadas e revisão da literatura de indicadores que cobriam preocupações em áreas diversas da vida. A primeira etapa compreendeu uma análise semântica dos itens, derivando um instrumento final composto por 27 deles que representavam as cinco dimensões das necessidades.

Leidy (1994) não checou a estrutura fatorial de sua medida. Entretanto, comprovou a consistência interna de cada uma das cinco dimensões hipotetizadas e evidências de sua validade de construto (ou seja, validade de critério, convergente). No caso, considerou duas amostras, sendo uma formada por 109 pessoas com doença pulmonar crônica e 100 saudáveis. Os coeficientes alfa de Cronbach variaram de 0,68 (necessidades fisiológicas) a 0,83 (necessidade de autorrealização), na primeira amostra $\left(\mathrm{M}_{\alpha}=0,76\right)$, e entre 0,61 (necessidade de estima) e 0,80 (necessidade de autorrealização), na segunda amostra $\left(M_{\alpha}=0,73\right)$. Como predito, as pessoas saudáveis pontuaram mais do que as enfermas nas dimensões de necessidades fisiológicas e de autorrealização; aquelas casadas o fizeram mais do que as solteiras na dimensão de afiliação; quem relatou contar com maior salário o fez na dimensão segurança; e aquelas com maior idade também o fizeram mais nesta dimensão de necessidade ( $p<0,01$ para todas as diferenças). Por fim, esta autora ainda observou que as necessidades maslownianas, todas admitidamente positivas, se correlacionaram inversamente com eventos estressantes na vida $\left(M_{r}=-0,40, p<0,001\right)$ e afetos negativos $\left(M_{r}=-0,43\right.$, $\mathrm{p}<0,001$ ), tendo-o feito positivamente com as medidas de autotranscedência $\left(M_{r}=0,41, p<0,001\right)$ e desenvolvimento psicossocial $\left(\mathrm{M}_{\mathrm{r}}=0,47, \mathrm{p}<0,001\right)$.

Diferentes estudos têm demonstrado a adequação do uso do ISNB para diversos fins. Por exemplo, foi empregado para examinar a relação entre a satisfação das necessidades, a alimentação emocional, as restrições dietética e as exceções de refeições (Cleary, \& Crafti, 2007), assim como para compreender os fatores que podem explicar a promoção de saúde e autocuidados (Acton, \& Malathum, 2000). Além disso, decidiu-se empregá-lo na Holanda para compreender o comportamento de escolha alimentar (Lenthe et al., 2015) e nos Estados Unidos, especificamente na população de Indiana, com o objetivo de avaliar o sentimento de pertença e o impacto na saúde mental (Hill, 2006). Em todos esses casos, este inventário se mostrou satisfatório para mensurar o que se propõe, isto é, a satisfação das necessidades humanas básicas, revelando-se promissor para explicar construtos que foram objetos de pesquisas empíricas. 
Em resumo, considerando o suporte teórico que fundamenta esta medida das necessidades maslownianas, seus parâmetros psicométricos e seu uso em diversas pesquisas, além da carência de instrumentos desta natureza no Brasil, decidiu-se realizar o esforço de adaptá-lo, reunindo evidências de sua validade fatorial, validade convergente e consistência interna. Os resultados a respeito podem ser observados nos estudos que são descritos a seguir, primeiramente realizando análises exploratórias e, posteriormente, procurando dirimir qualquer dúvida, checando a adequação da estrutura fatorial teorizada.

\section{Estudo 1. Evidências de validade fatorial e consistência interna da ISNB}

\section{Método}

Participantes

Este estudo contou com uma amostra não probabilística (de conveniência) formada por 200 estudantes universitários de instituição pública da cidade de João Pessoa (PB). Estes tinham idade média de 23,8 (DP $=6,89)$, sendo a maioria do sexo feminino $(56,5 \%)$, solteira $(84,5 \%)$ e de religião católica $(40,5 \%)$.

\section{Instrumentos}

Os participantes receberam um livreto/questionário com perguntas demográficas (estado civil, idade, religião e sexo) e duas medidas:

ISNB. Elaborado por Leidy (1994), fundamentando-se na tipologia das necessidades de Maslow, reúne 27 itens (por exemplo, Item 1. Como você se sente sobre o conforto físico de sua casa - temperatura, água, iluminação, ventilação; Item 12. Como você se sente sobre relações familiares próximas, como pais, sogros, irmãos e irmãs). Estes são respondidos em escala de sete pontos, variando de 1 (Totalmente insatisfeito) a 7 (Totalmente satisfeito). Informações sobre seus parâmetros psicométricos foram apresentadas previamente.

Escala de Satisfação com a Vida (ESV). Proposta originalmente por Diener, Emmons, Larsen e Griffin (1985), foi adaptada ao contexto brasileiro por Gouveia, Barbosa, Andrade e Carneiro (2005), os quais descrevem seus parâmetros psicométricos. Este instrumento é composto por cinco itens (por exemplo, Item 1. Eu me vejo como alguém que é conversador, comunicativo; Item 2. Eu me vejo como alguém que é minucioso, detalhista no trabalho), os quais são respondidos em escala de sete pontos, variando de 1 (Discordo totalmente) a 7 (Concordo totalmente).

Procedimento

Primeiramente, contataram-se os professores informando os objetivos do estudo e solicitando sua autorização para coletar os dados em suas salas, agendando previamente um horário de visita. Com sua anuência, procedeu-se à coleta dos dados, que aconteceu em contexto coletivo de sala de aula, porém os participantes responderam individualmente. Três colaboradores treinados ficaram responsáveis por esta atividade. Procurou-se assegurar o caráter anônimo do estudo, indicando-se que a participação das pessoas era voluntária. Após conhecerem os objetivos do estudo, eram solicitados a ler e assinar o Termo de Consentimento Livre e Esclarecido, indicando sua concordância em participar, autorizando que suas respostas pudessem ser consideradas, no conjunto, para fins científicos e/ou acadêmicos. A propósito, seguiram-se todas as recomendações éticas relacionadas às pesquisas com seres humanos, conforme Resolução no 512/2016, do Conselho Nacional de Saúde. O projeto obteve aprovação do Comitê de Ética em Pesquisa - Plataforma Brasil (Parecer no 883.309). O preenchimento do questionário durou, em média, quinze minutos.

Análise de Dados

Os dados foram analisados com o programa estatístico PASW (versão 18). Calcularam-se estatísticas descritivas, a exemplo de média e desvio padrão, que permitiram descrever os participantes do estudo, coeficientes de correlação de Pearson, Análise Multivariada de Variância (MANOVA), Análise Fatorial Exploratória e alfa de Cronbach, que favoreceram conhecer evidências de validade e consistência interna da medida ora adaptada.

\section{Resultados}

Inicialmente, procurou-se conhecer o poder discriminativo dos itens do ISNB. No caso, visando discriminar os participantes com magnitudes próximas no traço latente (necessidades), resolveu-se definir grupos-critério a partir da mediana de cada uma das cinco dimensões presumidas. Definidos os grupos inferior e superior, empregou-se a MANOVA para 
verificar a discriminação dos itens. Os resultados se mostraram adequados para as cinco dimensões, conforme resumidos a seguir.

A dimensão de necessidade fisiológica [Lambda de Wilks $=0,38$; $\mathrm{F}(6 / 193)=50,96, \mathrm{p}<0,001]$ apresentou tamanho de efeito $\left(h^{2}\right)$ de 0,62 . Todos os seus itens foram discriminativos $(\mathrm{p}<0,001)$, sendo os itens $18\left(h^{2}=0,20\right)$ e $19\left(h^{2}=0,33\right)$ aqueles com menor e maior poder discriminativo, respectivamente. Na mesma direção, os itens que compuseram a necessidade de segurança [Lambda de Wilks $=0,36$; $F(6 / 193)=57,29, \mathrm{p}<0,001]$ se revelaram discriminativos $\left(\eta^{2}=0,64\right)$, apresentando-se os itens $14\left(\mathrm{~h}^{2}=0,27\right)$ e $15\left(\mathrm{~h}^{2}=0,41\right)$ como os extremos de discriminação inferior e superior, respectivamente. A necessidade de afiliação mostrou resultados semelhantes [Lambda de Wilks $=0,37$; $\left.\mathrm{F}(5 / 193)=66,74, \mathrm{p}<0,001 ; \mathrm{h}^{2}=0,63\right]$, sendo que os itens $10\left(h^{2}=0,21\right)$ e $12\left(h^{2}=0,38\right)$ se relevaram o menos e o mais discriminativo, respectivamente. A necessidade de estima apresentou itens adequados quanto à discriminação [Lambda de Wilks $=0,38$; $\left.\mathrm{F}(4 / 193)=78,16 \mathrm{p}<0,001 ; \mathrm{h}^{2}=0,62\right]$, revelando-se os itens $7\left(h^{2}=0,31\right)$ e $8\left(h^{2}=0,47\right)$, respectivamente, como os de menor e maior discriminação. Por fim, a dimensão de necessidade de autorrealização [Lambda de Wilks = 0,35; F $(6 / 193)=58,50$, $\mathrm{p}<0,001]$ apresentou itens discriminativos, cujos efeitos mínimo e máximo corresponderam, respectivamente, aos itens $5\left(h^{2}=0,27\right)$ e $13\left(h^{2}=0,42\right)$. Em suma, todos os itens do ISNB podem ser considerados discriminativos, restando checar se é adequado admitir as cinco dimensões teorizadas.

Primeiramente, procurou-se conhecer a fatorabilidade da matriz de correlações entre os itens da medida. Neste caso, empregaram-se o Kaiser-Meyer-Olkin e o Teste de Esfericidade de Bartlett, tendo os resultados apoiado este tipo de análise: $\mathrm{KMO}=0,91 \mathrm{e}$ $\chi^{2}(351)=2.880,19, p<0,001$. Portanto, procedeu-se com uma Análise Fatorial Exploratória, adotando-se o método PAF (Principal Axis Factoring) e rotação varimax, fixando-se a extração de cinco fatores, coerente com o modelo proposto por Maslow. Entretanto, considerando o critério de Kaiser, isto é, valor próprio (eigenvalue) superior a 1, poderiam ser identificados até seis fatores. Porém, admitindo o critério de Cattell, pareceu razoável assumir os cinco fatores, o que foi feito, fixando saturação mínima de $|0,40|$ para constituição e interpretação do fator. Os resultados são mostrados na Tabela 1.

O primeiro fator foi denominado necessidade de autorrealização, sendo composto por seis itens (5, $8,9,13,22$ e 27), cujas saturações variaram de 0,41 (Item 8. Como você se sente sobre si mesmo) a 0,63 (Item 9. Como você se sente sobre a maneira como você lida com os problemas que surgem em sua vida), explicando $13,5 \%$ da variância total. Este apresentou consistência interna, isto é, alfa de Cronbach $(\alpha)$, de 0,86 .

$\mathrm{O}$ segundo fator, nomeado como necessidade de segurança, ficou formado por cinco itens $(1,4,6$, 15 e 23), cujas cargas fatoriais variaram de 0,40 (Item 1. Como você se sente sobre o conforto físico de sua casa - temperatura, água, iluminação, ventilação) a 0,65 (Item 15. Como você se sente sobre a sua segurança), explicando $10,5 \%$ da variância total. Seu alfa de Cronbach foi de 0,67 .

O terceiro fator reuniu três itens (17, 18 e 19), sendo definido como necessidade fisiológica; suas cargas fatoriais variaram de 0,55 (Item 17 . Como você se sente sobre a quantidade de amizade e do amor em sua vida) a 0,69 (Item 18. Como você se sente sobre sua vida sexual), contribuindo com a explicação de $10 \%$ da variância total. Seu alfa de Cronbach foi de 0,68 .

O quarto fator foi designado como necessidade de estima, constituindo-se de três itens (7, 10 e 11), cujas cargas fatoriais variaram de 0,60 (Item 10. O quanto você é aceito e incluído por outros) a 0,85 (Item 11. A forma como as pessoas te tratam). Este contribuiu com a explicação de 9,9\% da variância total, tendo alfa de Cronbach de 0,85.

O quinto e último fator foi denominado como necessidade de afiliação, agrupando três itens (3, 12 e 14), tendo suas cargas fatoriais variado de 0,54 (Item 14. A confiança das pessoas que você) a 0,72 (Item 3 . Sua vida familiar). Este fator explicou $8,6 \%$ da variância total, apresentado alfa de Cronbach de 0,79.

Por fim, procurando reunir evidências psicométricas adicionais, checou-se a validade convergente do ISNB com a ESV. No caso, os resultados mostraram que a satisfação com a vida se correlacionou significativa $(\mathrm{p}<0,001)$ e positivamente com os fatores de necessidade de autorrealização $(r=0,68)$, necessidade fisiológica $(r=0,44)$, necessidade de segurança $(r=0,43)$, necessidade de estima $(r=0,40)$ e necessidade de afiliação $(r=0,40)$. 
Tabela 1

Estrutura fatorial do ISNB.

\begin{tabular}{|c|c|c|c|c|c|}
\hline \multirow{2}{*}{ Itens Conteúdo } & \multicolumn{5}{|c|}{ Fatores } \\
\hline & 1 & 2 & 3 & 4 & 5 \\
\hline 09. A maneira como você lida com os problemas & 0,63 & 0,20 & 0,17 & 0,24 & 0,08 \\
\hline 05. A medida que desenvolve e amplia a vida & 0,59 & 0,03 & 0,27 & 0,21 & 0,14 \\
\hline 27. Sua vida como um todo & 0,58 & 0,28 & 0,30 & 0,33 & 0,31 \\
\hline 25. A medida de suas necessidades pessoais & 0,55 & 0,49 & 0,25 & 0,13 & 0,15 \\
\hline 24. A medida de sua realidade é consistente & 0,53 & 0,47 & 0,12 & 0,27 & 0,18 \\
\hline 26. A maneira como você gasta o tempo livre & 0,52 & 0,27 & 0,41 & 0,15 & 0,03 \\
\hline 13. As chances de desfrutar coisas agradáveis & 0,45 & 0,32 & 0,31 & 0,27 & 0,23 \\
\hline 22. Quão segura você está financeiramente & 0,45 & 0,36 & 0,22 & $-0,02$ & 0,22 \\
\hline 08. Em relação a si mesmo & 0,41 & 0,21 & 0,29 & 0,36 & 0,32 \\
\hline 04. A chance de conhecer outras pessoas & 0,35 & 0,04 & 0,28 & 0,34 & 0,23 \\
\hline 15. A sua segurança & 0,23 & 0,65 & 0,18 & 0,18 & 0,14 \\
\hline 06. O quão seguro você está em sua casa & 0,05 & 0,52 & 0,01 & 0,18 & 0,02 \\
\hline 23. O quão confiável as pessoas ao seu redor & 0,31 & 0,48 & 0,13 & 0,30 & 0,26 \\
\hline O conforto físico de sua casa & 0,11 & 0,41 & 0,19 & $-0,01$ & 0,36 \\
\hline 18. Sua vida sexual & 0,12 & 0,02 & 0,69 & 0,21 & 0,05 \\
\hline 17. A quantidade de amizade e amor na vida & 0,23 & 0,28 & 0,55 & 0,27 & 0,17 \\
\hline 20. A quantidade de diversão & 0,42 & 0,34 & 0,49 & 0,19 & 0,16 \\
\hline 19. Sua própria saúde e condição física & 0,29 & 0,08 & 0,47 & 0,11 & 0,30 \\
\hline 16. A sua criatividade & 0,24 & 0,29 & 0,45 & 0,09 & 0,06 \\
\hline Seu nível de atividade física & 0,32 & $-0,02$ & 0,35 & 0,01 & 0,19 \\
\hline 21. A qualidade do seu sono & 0,27 & 0,23 & 0,35 & $-0,04$ & 0,25 \\
\hline 11. A forma como as pessoas te tratam & 0,22 & 0,21 & 0,21 & 0,84 & 0,18 \\
\hline 07. A quantidade de respeito que recebe & 0,09 & 0,35 & 0,14 & 0,65 & 0,27 \\
\hline 10. O quanto você é aceito e incluído por outros & 0,34 & 0,17 & 0,14 & 0,59 & 0,17 \\
\hline Sua vida familiar & 0,17 & 0,02 & 0,03 & 0,16 & 0,72 \\
\hline 12. Relações familiares próximas & 0,14 & 0,14 & 0,09 & 0,26 & 0,67 \\
\hline 14. A confiança das pessoas que você depende & 0,12 & 0,43 & 0,23 & 0,27 & 0,53 \\
\hline Número de itens & 6 & 5 & 4 & 3 & 3 \\
\hline Valores próprios & 3,65 & 2,83 & 2,71 & 2,67 & 2,32 \\
\hline Variância explicada (\%) & 13,52 & 10,48 & 10,05 & 9,90 & 8,59 \\
\hline Alfa de Cronbach & 0,86 & 0,67 & 0,73 & 0,85 & 0,79 \\
\hline
\end{tabular}

\section{Discussão parcial}

O presente estudo teve como objetivo reunir as primeiras evidências de validade (fatorial e convergente) e precisão (consistência interna) do ISNB. Confia-se que este tenha sido alcançado, apresentando-se nesta oportunidade as primeiras evidências psicométricas que atesta a adequação desta medida no Brasil, fundamentada na teoria de Abraham Maslow. O presente estudo contribui, ainda, com a demonstração de evidências de validade fatorial desta medida; lembrando, em sua construção Leidy (1994) se pautou em validades convergente e discriminante, não apresentando qualquer resultado acerca de sua estrutura fatorial. Deste modo, o empreendimento aqui descrito realizou esforços que adicionam à literatura, além de contar por primeira vez com amostra de brasileiros. 
A teoria de Maslow, cuja estrutura das necessidades é dividida em cinco categorias, fundamenta diversos modelos teóricos e estudos empíricos (Gouveia, 2013; Tay, \& Diener, 2011). Entretanto, esta teoria ainda suscita discussões, sobretudo no que diz respeito ao conjunto de necessidades (Kenrick et al., 2010). Nesta direção, reunir evidências sobre a adequação do ISNB, que contempla os cinco fatores previstos nessa teoria, parece uma contribuição importante, sobretudo por explicar mais de $50 \%$ da variância total das respostas dos participantes, apresentando consistência interna (alfa de Cronbach) próxima ou superior ao que tem sido recomendado na literatura $(0,70$; Pasquali, 2010).

Apesar de os resultados apresentados suportarem a estrutura pentafatorial teorizada, os achados correspondentes se pautaram em análises exploratórias, sendo menos robustos. Esta indeterminação se fez presente nos próprios critérios de definição do número de fatores (por exemplo, Kaiser, Cattell). Fruto da natureza exploratória da análise realizada, alguns itens saturaram em fatores distintos aos que foram originalmente pensados. Estes aspectos motivaram o segundo estudo, que visou investigar a adequação do modelo com cinco fatores, tendo em conta análises fatoriais confirmatórias (Tabachnick, \& Fidell, 2013), confrontando-o com os modelos com um e seis fatores, que emergiu no estudo prévio.

\section{Estudo 2. Análise Fatorial Confirmatória do ISNB}

\section{Método}

Participantes

Contou-se com a participação de 199 estudantes universitários da cidade de Parnaíba (PI). Estes apresentaram idade média de 22,5 anos (variando de 18 a 45 anos; $\mathrm{DP}=5,99$ ), a maioria do sexo feminino $(77 \%)$.

Instrumento e procedimento

Os participantes responderam o ISNB, descrito no estudo anterior. Ademais, foram inseridas duas questões de natureza demográfica (idade e sexo). Adotou-se o mesmo procedimento descrito no Estudo 1, realizando a coleta em contexto coletivo, porém os participantes respondendo individualmente. Todos assinaram termo de consentimento livre e esclare- cido, sendo este estudo parte do projeto cuja aprovação no comitê de ética se especificou anteriormente.

Análise de dados

Dois pacotes estatísticos foram utilizados: PASW e AMOS (ambos na versão 18). O primeiro foi utilizado para calcular as estatísticas descritivas (medidas de tendência central e dispersão) e alfas de Cronbach; no caso do segundo, empregou-se para a realização das Análises Fatoriais Confirmatórias, comparando o modelo pentafatorial com os modelos alternativos: uni e hexafatorial. Para a realização destas análises, levou-se em conta a matriz de covariância como entrada, assumindo o estimador ML (Maximum Likelihood); foram considerados os seguintes indicadores de ajuste (Tabachnick, \& Fidell, 2013): (1) a razão c ${ }^{2} / g l$ (qui-quadrado/graus de liberdade) é um indicador que pondera a probabilidade de o modelo teórico se ajustar aos dados, recomendando-se valores entre 2 e 3, mas se admite até 5; (2) o Comparative Fit Index (CFI), que é um índice adicional de ajustamento, adotando o 0,90 como valor de referência para aceitação do modelo; e (3) o Root-Mean-Square Error of Approximation (RMSEA), cujos valores altos demonstram o mal ajustamento do modelo, recomendando-se aqueles entre 0,05 e 0,08, porém são admitidos até 0,10 como limite superior.

Por fim, verificaram-se três indicadores de comparação de modelos alternativos: o Expected Cross-Validation Index (ECVI), o Consistent Akaike Information Criterion (CAIC) e a diferença entre os qui-quadrados com seus respectivos graus de liberdade $\left[\Delta \mathrm{c}^{2} /(\mathrm{gl})\right]$. Os dois primeiros indicam o melhor modelo a partir da verificação dos menores valores, enquanto o último apresenta um nível significância para dizer se os modelos são estatisticamente diferentes, preferindo-se aquele com menor valor de $\mathrm{c}^{2}$.

\section{Resultados}

Inicialmente, realizou-se uma Análise Fatorial Confirmatória testando o modelo pentafatorial (Modelo 1), considerando-se a estrutura do primeiro estudo. Os resultados mostraram que o item 6 (O quão seguro você está de pessoas que poderiam roubar ou destruir sua casa) não apresentou saturação (lambda) estatisticamente diferente de zero $(\lambda \neq 0 ; \mathrm{z}<1,96, \mathrm{p}>0,05)$, porém todos os demais o fizeram. Com isso, procedeu-se com uma nova análise, excluindo o item 6 , observando-se os seguintes indicadores de ajuste: $\chi^{2}(142)=286,297$, 
$\mathrm{p}<0,001 ; \chi^{2} / \mathrm{gl}=2,02 ; \mathrm{CFI}=0,90$ e RMSEA $=0,07$ (IC90\% $=0,059-0,083)$. A estrutura fatorial correspondente é mostrada na Figura.

Posteriormente, com o fim de averiguar a pertinência de considerar modelos alternativos para explicar a estrutura das necessidades, testaram-se os modelos unifatorial e hexafatorial. O primeiro define que todos os itens podem ser explicados por um único fator geral de necessidade, primando, portanto, pela parcimônia do modelo (Modelo 2), e o segundo, hexafatorial, considera a estrutura encontrada no Estudo 1 a partir do critério de Kaiser (Modelo 3). Os resultados destas análises são sumarizados na Tabela 2.

De acordo com os resultados da Tabela 2, os modelos com cinco (Modelo 1) e seis (Modelo 3) fatores se mostraram os melhores, reunindo indica-

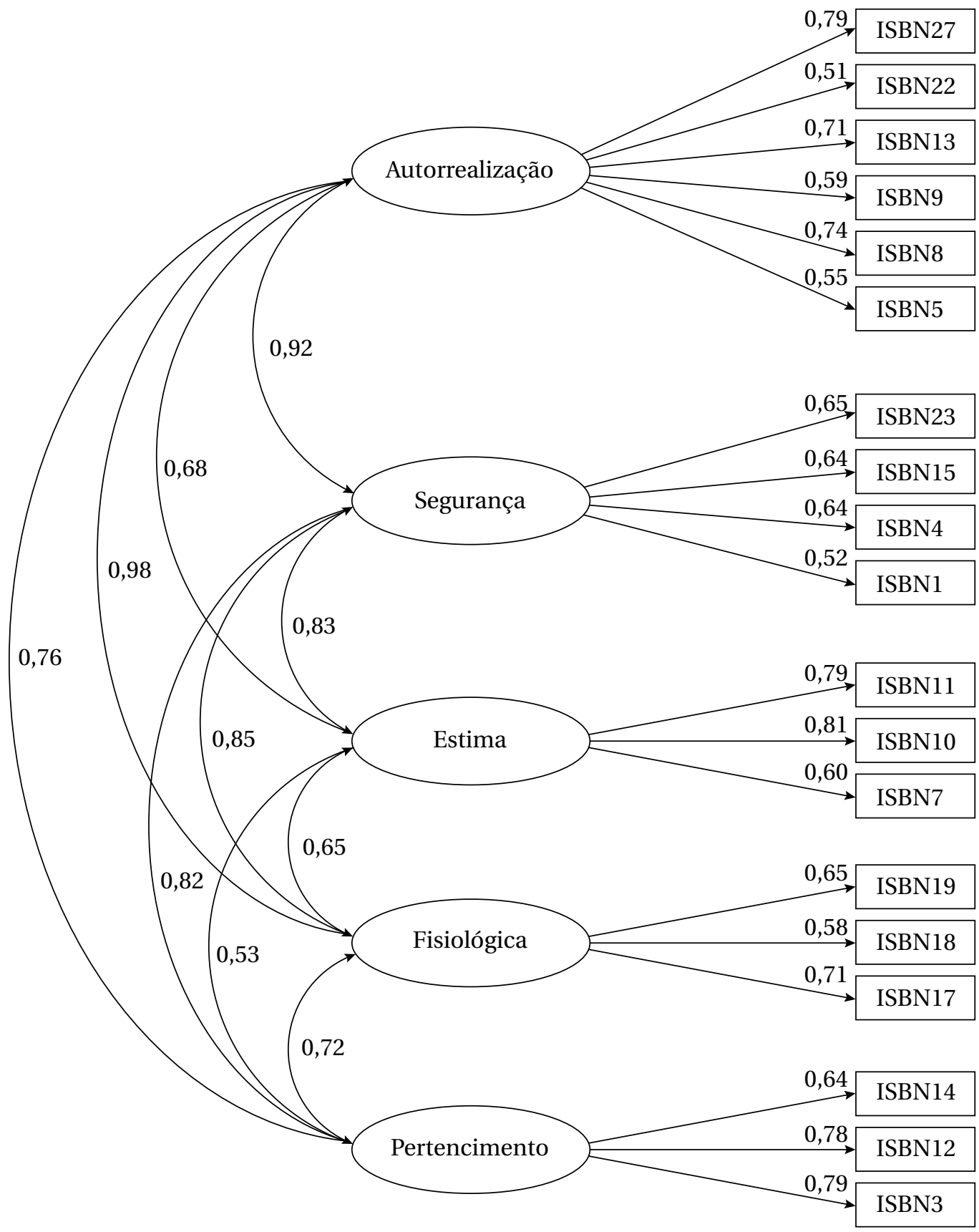

Figura

Estrutura fatorial do Inventário de Satisfação das Necessidades Básicas. 
Tabela 2

Comparação de diferentes modelos fatoriais da medida de satisfação das necessidades.

\begin{tabular}{lccccccc}
\hline Modelo & $\mathrm{c}^{2}(\mathrm{gl})$ & $\mathrm{c}^{2} / \mathrm{gl}$ & CFI & RMSEA (IC90\%) & ECVI & CAIC & Dc $^{2}(\mathrm{gl})$ \\
\hline 1 & $320,50(160)$ & 2,02 & 0,90 & 0,07 & 1,92 & 588,52 & - \\
2 & $449,81(170)$ & 2,64 & 0,82 & 0,09 & 2,67 & 701,55 & $129,31(10)^{*}$ \\
3 & $368,43(194)$ & 1,89 & 0,90 & 0,06 & 2,45 & 739,74 & $47,94(34) \dagger$ \\
\hline
\end{tabular}

Error of Approximation (IC90\% = Intervalo de Confiança de 90\%).

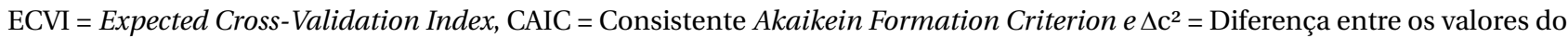
qui-quadrado.

${ }^{*} \mathrm{p}<0,001 \mathrm{e}+\mathrm{p}<0,10$.

Modelo 1 = Pentaforial, Modelo $2=$ Unifatorial e Modelo $3=$ Hexafatorial.

dores de ajuste mais satisfatórios do que os observados para o Modelo 2 (unifatorial). Entretanto, parece mais promissor o modelo proposto originalmente por Maslow, ou seja, com cinco fatores, que se mostrou marginalmente superior ao modelo hexafatorial $\left[\Delta c^{2}(34)=47,93, p=0,057\right]$. Reforçam esta evidência os índices ECVI e CAIC, que no modelo pentafatorial foram claramente inferiores aos hexafatorial. Por fim, calcularam-se os alfas de Cronbach para cada um dos cinco fatores teorizados, observando-se os seguintes coeficientes: fisiológicas $(\alpha=0,69)$, segurança $(\alpha=0,70)$, afiliação $(\alpha=0,77)$, estima $(\alpha=0,77)$ e autorrealização $(\alpha=0,81)$.

\section{Discussão Parcial}

Este estudo objetivou investigar a estrutura pentafatorial proposta por Leidy (1994), consoante com o modelo de Maslow (1954), confrontando-a com os modelos alternativos uni e hexafatorial. Percebeu-se que os modelos com cinco e seis fatores se mostraram mais ajustados, sendo que aquele teorizado foi ainda mais promissor. Apesar de o modelo original ter apresentado, no geral, melhor ajuste que os demais, é preciso interpretar com ponderação este achado. Primeiro, contou-se com uma amostra de conveniência (não probabilística), composta unicamente por estudantes universitários. Segundo, Leidy (1994) não testou a estrutura teorizada na versão original de seu instrumento, conhecendo apenas evidências de suas validades convergente e discriminante, além da consistência interna de seus fatores presumidos. Neste sentido, este é o primeiro estudo a oferecer evidências a respeito, carecendo de replicações em outros contextos e considerando outros participantes. Além disso, será preciso avaliar outros aspectos, a exemplo da invariância fatorial, o que permitirá assegurar que a estrutura não varia em função de pertença a grupos ou características demográficas.

\section{Discussão e considerações futuras}

O objetivo principal deste artigo foi adaptar o ISNB, reunindo evidências de sua validade (fatorial e convergente) e consistência interna. Trata-se de uma medida que avalia necessidades humanas a partir do modelo teórico proposto por Maslow (1943), que tem sido empregado em estudos prévios (Acton, \& Malathum, 2000; Cleary, \& Crafti, 2007; Hill, 2006; Lenthe et al. 2015), porém não se encontrou qualquer estudo, incluindo no Brasil, que tivesse investigado sua estrutura fatorial, inclusive em seu estudo de elaboração (Leidy, 1994). Deste modo, confia-se ter alcançado o objetivo proposto, oferecendo contribuição relevante à literatura sobre necessidades e construtos correlatos.

Em termos gerais, observou-se que os itens desta medida reuniram parâmetros psicométricos adequados, tendo seus itens se revelado discriminativos (Pasquali, 2010). A análise fatorial exploratória (Estudo 1), embora inconclusiva, sugerindo cinco (critério de Cattell) ou seis (critério de Kaiser) fatores, encontrou respaldo com a análise fatorial confirmatória (Estudo 2), ocasião em que a proposta de Maslow (1943) pareceu encontrar mais respaldo. Isso, certamente, justifica o fato de este modelo com cinco dimensões ser recorrente na literatura (Acton, \& Malathum, 2000; Taormina, \& Gao, 2013; Tay, \& Diener, 2011). Os indicadores de ajuste na análise fatorial confirmatória, embora não perfeitos, foram muito promissores (por exemplo, $\mathrm{CFI}=0,90, \mathrm{RMSEA}<0,08$ ), atendendo ao que se costuma recomendar na literatura (Tabachnick, \& 
Fidell, 2013), favorecendo pensar em um instrumento com estrutura pentafatorial consoante com o que previa Maslow (1954).

A propósito ainda do parâmetro de validade, considerou-se em que medida as necessidades poderiam estar correlacionadas com a satisfação com a vida. No caso, consoante com os achados de Leidy (1994), que observou correlações positivas na casa de 0,40 com medidas de autotranscedência e desenvolvimento psicossocial, neste artigo (Estudo 1) as correlações das necessidades com aquela variável de bem-estar subjetivo foram iguais ou superiores a 0,40 , alcançando 0,68 para a necessidade de autorrealização. Isso é coerente com a concepção humanista maslowniana, que defende que todas as necessidades são positivas e, quando realizadas, promovem a saúde mental e o bem-estar psicológico (Maslow, 1954).

Quanto às evidências de precisão desta medida, isto é, de sua consistência interna, os coeficientes observados foram dentro do que se recomenda na literatura (Pasquali, 2010), sendo justificável seu uso no contexto de pesquisa. Ressalta-se que se trata de um instrumento breve (menos de 30 itens), tipo lápis e papel, de fácil aplicação e avaliação, representando uma contribuição prática para conhecer as necessidades das pessoas em contexto brasileiro ou, inclusive, identificar os antecedentes e consequentes deste construto. A propósito, sua relação tem sido presumida com as necessidades (Gouveia, 2013), porém, em termos empíricos, pouco ainda se sabe, abrindo-se uma possibilidade para estudos futuros a respeito.

A propósito de estudos futuros, a agenda parece abrangente. Será preciso, além de checar a adequação desta medida em outros grupos, avaliar novos parâmetros psicométricos. Nesta direção, caberá investigar, por exemplo, sua estabilidade temporal (teste-reteste) e avaliar evidências de sua validade discriminante, por exemplo. Neste caso, talvez o primeiro a fazer será avaliar em que medida suas pontuações estão isentas de desejabilidade social, um atributo que poderá afetar os resultados da pesquisa sobretudo para atributos positivos (Gouveia, Guerra, Sousa, Santos, \& Costa, 2009), como podem ser essas necessidades.

Por fim, apesar de os achados anteriormente descritos serem favoráveis ao ISNB, não se descartam limitações dos estudos levados a cabo. Como já se anunciou, ambos contaram com a participação de estudantes universitários, que compuseram amostras de conveniência. Isso não permite generalizar os achados sobre grau de satisfação das necessidades das pessoas no Brasil, nem mesmo na cidade de João Pessoa. Contudo, tenha-se em conta que não foi este o propósito desses estudos, que tiveram cunho eminentemente psicométrico, focado na medida em si.

Nesta direção, teve-se em conta o marco da Teoria Clássica dos Testes, que compreende uma via legítima para os propósitos dos estudos descritos (Pasquali, 2010). Não obstante, recomendam-se também estudos que tenham em conta a Teoria de Resposta ao Item, que poderão complementar evidências de adequação do conjunto de itens deste instrumento. Em qualquer caso, confia-se ter oferecido nesta oportunidade um instrumento que reúne dois dos parâmetros psicométricos essenciais [validade (fatorial e convergente) consistência interna], que dão conta da adequação desta medida, que poderá ser empregada em contexto de pesquisa.

\section{Referências}

Acton, G. J., \& Malathum, P. (2000). Basic need status and health-promoting self-care behavior in adults. Western Journal of Nursing Research, 22(7), 796-811. https://doi.org/10.1177/01939450022044764

Andrews, F. M., \& Withey, S. B. (1974). Developing measures of perceived life quality: Results from several national surveys. Social Indicators Research, 1(1), 1-26. https://doi.org/10.1007/BF00286419

Carretié, L., Hinojosa, J. A., Mercado, F., \& Tapia, M. (2005). Cortical response to subjectively unconscious danger. Neuroimage, 24(3), 615-623. https:// doi.org/10.1016/j.neuroimage.2004.09.009

Cleary, J. M. \& Crafti, N. (2007). Basic need satisfaction, emotional eating, and dietary restraint as risk factors for recurrent overeating in a community sample. Sensoria: A Journal of Mind, Brain \& Culture, 3(2), 27-39. https:// doi.org/10.7790/ejap.v3i2.90

Diener, E., Emmons, R. A., Larsen, R. J., \& Griffin, S. (1985). The satisfaction with life. Journal of Personality Assessment, 49(1), 71-75. https://doi.org/10.1207/s15327752jpa4901_13 
Furnham, A. (2005). The psychology of behaviour at work: The individual in the organization. London: Psychology.

Gouveia, V. V. (2013). Teoria funcionalista dos valores humanos: Fundamentos, aplicações e perspectivas. São Paulo, SP: Casa do Psicólogo.

Gouveia, V.V., Barbosa, G. A., Andrade, E. D. O., \& Carneiro, M. B. (2005). Medindo a satisfação com a vida dos médicos no Brasil. Jornal Brasileiro de Psiquiatria, 54(4), 298-305.

Gouveia, V. V., Guerra, V. M., Sousa, D. M. F. D., Santos, W. S., \& Costa, J. D. M. (2009). Escala de desejabilidade social de Marlowe-Crowne: Evidências de sua validade fatorial e consistência interna. Avaliação Psicológica, 8(1), 87-98.

Hill, D. L. (2006). Sense of belonging as connectedness, american indian worldview, and mental health. Archives of Psychiatric Nursing, 20(5), 210-216.

Kenrick, D. T., Griskevicius, V., Neuberg, S. L., \& Schaller, M. (2010). Renovating the pyramid of needs contemporary extensions built upon ancient foundations. Perspectives on Psychological Science, 5(3), 292-314. https://doi. org/10.1177/1745691610369469

Kellerman, A. (2014). The satisfaction of human needs in physical and virtual spaces. The Professional Geographer, 66(4), 538-546. https://doi.org/10.1080/00330124.2013.848760

Leidy, N. K. (1994). Operationalizing Maslow's theory: Development and testing of the basic need satisfaction inventory. Issues in Mental Health Nursing, 15(3), 277-295. https://doi.org/10.3109/01612849409009390

Lenthe, F. J., Jansen, T., \& Kamphuis, C. (2015). Understanding socio-economic inequalities in food choice behaviour: Can Maslow's pyramid help? British Journal of Nutrition, 113(7), 1139-1147. http://doi.org/10.1017/ S0007114515000288

Lester, D. (2013). Measuring maslow's hierarchy of needs. Psychological Reports, 131(1), 15-17. https://doi. org/10.2466/02.20.PR0.113x16z1

MacDonald, G. \& Leary, M. R. (2005). Why does social exclusion hurt? The relationship between social and physical pain. Psychological Bulletin, 131(2), 202-223. https://doi.org/ 10.1037/0033-2909.131.2.202

Maslow, A. H. (1943). A theory of human motivation. Psychological Review, 50(4), 370-396. https://doi. org/10.1037/h0054346

Maslow, A. H. (1954). Motivation and personality. New York, NY: Harper

Pasquali, L. (2010). Instrumentação psicológica: Fundamentos e práticas. Porto Alegre, RS: Artmed.

Tabachnick, B. G. \& Fidell, L. S. (2013). Using multivariate statistics (6a ed.). Boston: Allyn and Bacon.

Taormina, R. J. \& Gao, J. H. (2013). Maslow and the motivation hierarchy: Measuring satisfaction of the needs. The American Journal of Psychology, 126(2), 155-177. https://doi.org/10.5406/amerjpsyc.126.2.0155

Tay, L. \& Diener, E. (2011). Needs and subjective well-being around the world. Journal of Personality and Social Psychology, 101(2), 354-365. https://doi.org/10.1037/a0023779

Yang, K. S. (2003). Beyond Maslow's culture-bound linear theory: A preliminary statement of the double-y model of basic human needs. In V. Murphy-Berman, \& J. J. Berman (Eds.), Cross-cultural differences in perspectives on the self (pp. 192-272). Lincoln, NE: University of Nebraska.

\section{Thiago Medeiros Cavalcanti}

Doutor pela Universidade Federal da Paraíba (UFPB), João Pessoa - PB. Brasil.

E-mail: thiagomcavalcantii@gmail.com

(iD http://orcid.org/0000-0003-4554-7539

\section{Valdiney Veloso Gouveia}

Doutor pela Universidad Complutense de Madrid, Espanha.

E-mail:vvgouveia@gmail.com

(iD http:// orcid.org/0000-0003-2107-5848 


\section{Emerson Diógenes de Medeiros}

Doutor pela Universidade Federal da Paraíba (UFPB), João Pessoa - PB. Brasil.

E-mail: mersondiogenes@gmail.com

(iD) http://orcid.org/0000-0002-1407-3433

\section{Tailson Evangelista Mariano}

Doutorando da Universidade Federal da Paraíba (UFPB), João Pessoa - PB. Brasil.

E-mail: tailsonmariano@hotmail.com.br

(iD) http://orcid.org/0000-0001-6716-0250

\section{Hysla Magalhães de Moura}

Doutoranda da Universidade do Estado do Rio de Janeiro (UERJ), Rio de Janeiro - RJ. Brasil.

E-mail: hyslamagalhaes@gmail.com

(iD) http://orcid.org/0000-0002-5866-5799

\section{Heloísa Bárbara Cunha Moizeís}

Psicóloga pela Universidade Federal da Paraíba (UFPB), João Pessoa - PB. Brasil.

E-mail: heloisabarbara96@gmail.com

(iD http://orcid.org/0000-0003-0477-8410

Endereço para envio de correspondência:

Universidade Federal da Paraíba, CCHL - Departamento de Psicologia. CEP: 58051 - 900. João Pessoa - PB. Brasil.

Recebido $31 / 07 / 2017$

Reformulado 25/04/2018

Aceito 04/07/2018

Received $07 / 31 / 2017$

Reformulated $04 / 25 / 2018$

Approved 07/04/2018

Recibido 31/07/2017

Reformulado $25 / 04 / 2018$

Aceptado 04/07/2018

Como citar: Cavalcanti, T. M., Gouveia, V.V., Medeiros, E. D., Mariano, T. E., Moura, H. M., Moizéis, H. B. C. (2019). Hierarquia das necessidades de Maslow: Validação de um Instrumento. Psicologia: Ciência e Profissão, 39, 1-13. https://doi.org/10.1590/1982-3703003183408

How to cite: Cavalcanti, T. M., Gouveia, V.V., Medeiros, E. D., Mariano, T. E., Moura, H. M., Moizéis, H. B. C. (2019). Maslow's Hierarchy of Needs: Validation of an Instrument. Psicologia: Ciência e Profissão, 39, 1-13. https://doi.org/10.1590/1982-3703003183408

Cómo citar: Cavalcanti, T. M., Gouveia, V.V., Medeiros, E. D., Mariano, T. E., Moura, H. M., Moizéis, H. B. C. (2019). Jerarquía de Necesidades de Maslow: Validación de un Instrumento. Psicologia: Ciência e Profissão, 39, 1-13. https://doi.org/10.1590/1982-3703003183408 